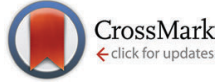

Cite this: Phys. Chem. Chem. Phys., 2014, 16, 22723

Received 12th July 2014, Accepted 8th August 2014

DOI: $10.1039 / c 4 c p 03068 k$

www.rsc.org/pccp

\section{Enhanced catalytic and SERS activities of CTAB stabilized interconnected osmium nanoclusters $\dagger$}

\author{
Sivasankara Rao Ede, U. Nithiyanantham and Subrata Kundu*
}

A new route for the formation of osmium nanoparticles (NPs) having different morphologies like aggregated clusters, chain-like networks, and small spheres are reported. The synthesis was done by utilizing a simple wet-chemical method at room temperature (RT) by the reaction of $\mathrm{OsO}_{4}$, cetyl trimethyl ammonium bromide (CTAB), 2,7-dihydroxynaphthalene (2,7-DHN) and $\mathrm{NaOH}$ under 30 min of reaction. The diameter of the individual particles in all the morphologies was $\sim 1-3 \mathrm{~nm}$. The synthesized materials have been tested for catalysis and SERS studies. The catalysis study was examined taking different organic nitro compounds and the catalysis rate was found superior as compared to other reports. The surface enhanced Raman scattering (SERS) study was done taking Rose Bengal (R Be) as a probe molecule and the observed enhancement factor (EF) value was found superior or comparable to most of other noble metal NPs. The overall synthesis process was simple, less time consuming and cost-effective. The enhanced catalytic and SERS activities of the Os NCS might open up a new avenue for the application in other organic catalysis reactions, SERS based detection of environmentally important trace bio-molecules and sensors.

\section{Introduction}

In last few years the study on materials mainly at nanoscale dimensions found tremendous importance due to their unusual electronic, ${ }^{1}$ catalytic, ${ }^{2}$ magnetic, ${ }^{3}$ optical properties ${ }^{4}$ as compared to their bulk counterparts. So a burst of research activities has been seen in recent years for the synthesis and characterization of metal nanoparticles (NPs) and their possible application in different interdisciplinary fields. ${ }^{2,5,6}$ Metal NPs of varying sizes can be prepared by physical as well as chemical methods and the organization of metal NPs with a fixed number of atoms assumes significance. ${ }^{7}$ The potential use and rapid assembly of nanomaterials and organics have been reviewed by Varma et al. recently. ${ }^{8}$ Medintz et al. observed the possibility of functionalizations of NPs with different bio-molecules for various applications. ${ }^{9}$ The surface functionalized NPs can be used to bridge the gap between heterogeneous and homogeneous catalysis. ${ }^{10}$ Among different noble metals like $\mathrm{Au}, \mathrm{Ag}, \mathrm{Pd}, \mathrm{Pt}, \mathrm{Cu}, \mathrm{Ir}, \mathrm{Rh}, \mathrm{Ru}$ and Os, the study on osmium metal NPs has found very limited

Electrochemical Materials Science (ECMS) Division, CSIR-Central Electrochemical Research Institute (CECRI), Karaikudi-630006, Tamil Nadu, India.

E-mail: skundu@cecri.res.in, subrata_kundu2004@yahoo.co.in;

Fax: +91-4565-227651; Tel: +91-4565-241487

$\dagger$ Electronic supplementary information (ESI) available: Instruments used, the discussions related to sample preparation for various spectroscopic characterizations, EDS analysis, a study with other reaction parameters and a catalysis study are given. Figures related to EDS, XRD analysis, FT-IR study, TEM images for control experiments, UV-Vis spectra for the catalysis study, schemes and tables related to synthesis and catalysis studies are provided. See DOI: 10.1039/c4cp03068k application. Osmium is an interesting noble metal which competes with iridium and has very low compressibility, a high melting point and high bulk modulus. ${ }^{11}$ Due to many properties of Os and its oxide, Os metal is a demanding material to manufacture and work with. There are few reports available where Os particles were prepared in the form of thin films or sometimes they combined with other metals like Pd or Pt using mostly physical vapour deposition (PVD) routes. ${ }^{12-15}$ The application of Os thin films appears in the patent literature only related to the fields of fuel cells, catalysis, sensors and electronic devices. Os metal is usually alloyed with other metals for different uses such as instrument pivots, electrical contacts, fountain pen tips or others. Os doped $\mathrm{SnO}_{2}$ has been used for methane gas sensing ${ }^{16}$ while Os-Pd NPs on carbon nanotubes have been used for catalysis. ${ }^{14}$ Os NPs are also used as anode materials in direct borohydride fuel cells. ${ }^{17}$ Os electrocatalyst has been used for oxygen reduction reaction (ORR) in presence or in absence of aqueous methanol. ${ }^{18}$ Kramer and co-workers reported the synthesis of iron, ruthenium and osmium NPs from their metal carbonyl precursors. ${ }^{19}$ Recently, Os thin films have been grown by an atomic layer deposition route at higher temperature from an osmocene salt precursor. $^{20}$ Most of the above reports deal with the preparation of Os particles in the form of thin films where controlling the particle size and shape is rather difficult and the process needs high temperature and hard work to get the desired materials. The wet chemical synthesis of Os metal NPs in the literature is very limited. ${ }^{21,22}$ Recently, Chakrapani and Sampath reported the synthesis of small Os NPs by ascorbic acid reduction at $95{ }^{\circ} \mathrm{C} .{ }^{21}$ Very recently, we synthesized wire-like and honey-comb like Os 
nanoclusters (NCs) using deoxyribonucleic acid (DNA) as a scaffold using microwave heating. ${ }^{22}$ So, the synthesis of Os NPs or NCs using low temperature and a cost effective route is extremely desired.

It is well-known that metal NPs can be used as a good catalyst in various types of catalysis reactions due to their high surface to volume ratio and that the catalytic efficiency strongly depends on their sizes and shapes. The reduction of aromatic nitro groups using excess $\mathrm{NaBH}_{4}$ in the presence of metal NPs as catalyst has been investigated. ${ }^{2,23-25}$ Very recently, shapeselective Au-Ag core-shell nanocrystals have been utilized for catalytic reduction of nitro compounds. ${ }^{26}$ Apart from the catalysis reaction, metal NPs were also tested for surface enhanced Raman scattering (SERS) studies. ${ }^{27-30}$ In SERS, the plasmonic coupling effect at the nanometric gap junctions between the particles induces an enormous electromagnetic enhancement that allows the SERS signal to be detected with single molecular sensitivity. Moreover, SERS enables molecules to be identified by their vibrational 'fingerprint' and the other applications range from detection of bio-molecules, sensors or even in medical therapeutics. As the surface plasmon resonance (SPR) band of Os metal falls in the visible region like $\mathrm{Au}, \mathrm{Ag}$ etc., it is assumed that it can also be used as a potential substrate in a SERS study and in catalysis reactions. In recent reports as described above, ${ }^{21,22}$ authors observed that Os NPs can be used as potential SERS substrate while they used Rhodamine 6 G (R6G) and Methylene Blue (MB) as a potential SERS probe.

In the present report, we put forward a new and novel methodology for the room temperature (RT) synthesis of interconnected osmium nanoclusters within $30 \mathrm{~min}$ of a wet chemical reaction. The Os NPs having different morphologies like Os NCs, Os chain-like networks or ultrasmall nano spheres have been formed for the first time by the reaction of $\mathrm{OsO}_{4}$ with cetyl trimethyl ammonium bromide (CTAB) in presence of alkaline 2,7-dihydroxynaphthalene (2,7-DHN) under continuous stirring for just $30 \mathrm{~min}$. The size and morphology of the particles can be tuned just by controlling the surfactant to metal ion molar ratio and altering the other reaction parameters. The eventual diameters of the individual particles range between $\sim 1-3 \mathrm{~nm}$. The catalytic activity of the nanostructures has been tested for the reduction of 4-nitroaniline (4-NA) with $\mathrm{NaBH}_{4}$ in aqueous solution. The SERS activity of the Os nanostructures was examined using Rose Bengal ( $\mathrm{R} \mathrm{Be}$ ) as a model dye molecule. The present synthesis process is simple, reproducible and completed within a $30 \mathrm{~min}$ timescale. The self-assembled Os nanostructures generate a highly stable and reproducible SERS signal. The fast generation of CTAB stabilized Os NCs having a different morphology and their superior catalytic and SERS activity has not been looked at before.

\section{Experimental}

\section{Reagents and instruments}

Cetyl trimethyl ammonium bromide (CTAB, 99\%), 2,7-dihydroxynaphthalene $(2,7-\mathrm{DHN})$, osmium tetraoxide $\left(\mathrm{OsO}_{4}\right)$ and sodium hydroxide $(\mathrm{NaOH})$ were purchased from Sigma-Aldrich and used as received. Different nitro compounds like 4-nitroaniline (4-NA) was purchased from S D Fine Chemicals limited, Mumbai and others like 2-nitroaniline (2-NA), 4-nitrophenol (4-NP) and 2-nitrophenol (2-NP) were purchased from Sigma-Aldrich, Bangalore and used as received. Sodium borohydride $\left(\mathrm{NaBH}_{4}\right)$ was also purchased from Sigma-Aldrich and used freshly daily during the catalysis study. The dye molecule, Rose Bengal ( $\left.\mathrm{R} \mathrm{Be}, \mathrm{C}_{20} \mathrm{H}_{4} \mathrm{Cl}_{4} \mathrm{I}_{4} \mathrm{O}_{5}\right)$ was purchased from Qualigens Fine Chemicals, Mumbai. De-ionized (DI) water was used for the entire synthesis process. The synthesized CTAB-Os NCs were characterized with several spectroscopic techniques like UV-Vis, TEM, EDS, XRD and FT-IR analysis and their detailed specifications including a surface enhanced Raman scattering (SERS) study are given in the ESI. $\dagger$

\section{Synthesis of different morphologies of CTAB-Os NCs}

Os NCs having different morphologies have been synthesized by the reaction of $\mathrm{OsO}_{4}$ with $\mathrm{CTAB}$ and alkaline conditions in the presence of 2,7-DHN. In a typical reaction, $20 \mathrm{~mL}$ of stock CTAB solution $\left(10^{-2} \mathrm{M}\right)$ was mixed with $2 \mathrm{~mL}$ of $\mathrm{OsO}_{4}$ solution $\left(10^{-2} \mathrm{M}\right)$ and stirred well for homogeneous mixing. Then $1 \mathrm{~mL}$ of freshly prepared 2,7-DHN solution was added and stirred for another $5 \mathrm{~min}$. Finally, $200 \mu \mathrm{L}$ of $\mathrm{NaOH}$ solution $\left(10^{-2} \mathrm{M}\right)$ was added and the stirring was continued for another 10-15 min. The overall reaction time was $\sim 30 \mathrm{~min}$. The solution was initially colorless but a light pale violet color appears after addition of $\mathrm{NaOH}$. The pale violet color becomes dark with increasing the time and after $10 \mathrm{~min}$ the solution became darkviolet. The resultant solution contains Os NPs with an aggregated cluster like morphology. The solution was centrifuged 3-4 times at $8000 \mathrm{rpm}$ to remove the excess surfactant and other impurities and finally re-dispersed in DI water. The Os NPs solutions became ready for characterization and application purposes. The other sets were also prepared by changing the molar ratio of Os salt to $\mathrm{CTAB}$ and changing the other reaction parameters. The detailed concentration of all the reagents, reaction time, particle size and morphologies are given in Table 1. The synthesized Os particles were found very stable for more than six months while stored in a refrigerator in a sealed bottle without altering any optical properties. The CTAB-Os NPs were characterized using various spectroscopic tools and the details of the sample preparation procedures are given in the ESI. $\dagger$

\section{Catalytic reduction of 4-nitro aniline (4-NA) using CTAB-Os NCs as catalyst}

The catalytic reduction of aromatic nitro compounds was tested with CTAB-Os NCs as catalyst for the first time. The entire catalysis reduction was done with 4-NA as a specific example although several other nitro compounds were also examined. Among the different morphologies prepared, an Os aggregated cluster-like structure was used in a detailed catalysis study, although other morphologies were also tested for comparison purposes. For a typical catalysis reaction, $4 \mathrm{~mL}$ of DI water was mixed with $500 \mu \mathrm{L}$ of $\left(10^{-3} \mathrm{M}\right)$ stock 4-NA solution and stirred for $5 \mathrm{~min}$ for homogeneous mixing. After that, $1 \mathrm{~mL}$ of $0.1 \mathrm{M}$ ice-cold $\mathrm{NaBH}_{4}$ solution was added and shaken well. Finally, $400 \mu \mathrm{L}$ of Os aggregated cluster solution was added and the reaction was monitored using an UV-Vis spectrophotometer 
Table 1 Detailed final concentration of all the reagents, reaction time, average particles size and shapes

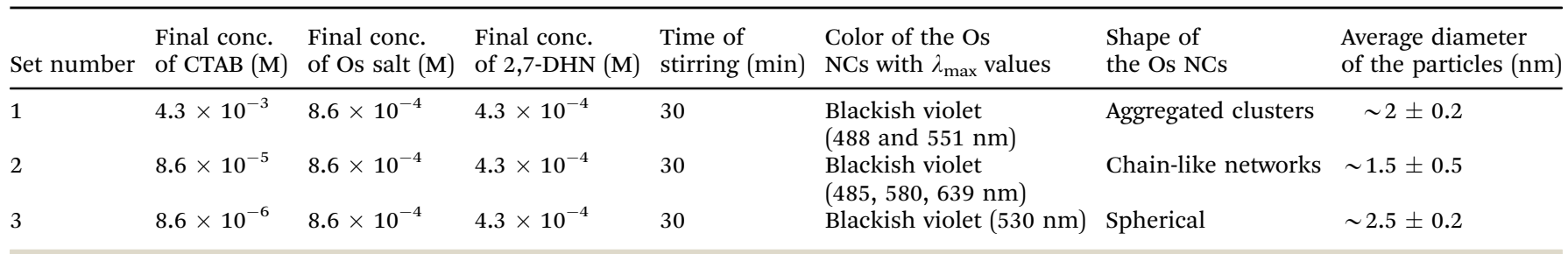

over different time intervals. The absorption spectra were recorded every 1-2 min until completion of the reduction process. The total time required for the reduction was $\sim 10 \mathrm{~min}$ and the light yellowish 4-NA solution turned colorless due to the formation of the reduced product para-phenylenediamine ( $p$-PDA) in the reaction medium. The completion of the reaction was confirmed by a change in the color of the solution as well as from the absorption bands in the UV-Vis spectrum.

\section{Results and discussion}

\section{UV-Vis spectroscopic study}

The Os NPs with different morphologies were synthesized by reduction of $\mathrm{OsO}_{4}$ in the presence of alkaline 2,7-DHN at RT under continuous stirring for $\sim 30 \mathrm{~min}$. Fig. 1A shows the UV-Vis spectra at different stages of the synthesis process. The colorless aqueous solution of CTAB has no specific absorption bands as seen in curve a, Fig. 1A. An aqueous solution of 2,7-DHN has two distinct absorption bands at 230 and $283 \mathrm{~nm}$ due to the presence of an aromatic benzene ring in its skeleton (curve b, Fig. 1A). Only an aqueous salt solution of $\mathrm{OsO}_{4}$ has an absorption maxima at $245 \mathrm{~nm}$ with a small hump at $279 \mathrm{~nm}$ due to ligand to metal charge transfer (LMCT) spectra (curve c, Fig. 1A). Similar types of LMCT spectra were observed for Au salts during preparation of Au NPs. ${ }^{31}$ A mixture of CTAB with Os salts deals with similar types of absorption profile although slight reduction of the absorbance value with a small shifting of the band maxima as compared to Os salt alone was observed (curve d, Fig. 1A) due to interaction of Os salt with CTAB. Now, just after mixing of alkaline 2,7-DHN with CTAB and Os salt, a new band at $327 \mathrm{~nm}$ was observed including the original 2,7-DHN band at $230 \mathrm{~nm}$ as seen in curve e, Fig. 1A. Now with increasing reaction time and continuous stirring, the clear solution became faintly violet (indicating nucleating started) initially, then light violet and finally dark violet after 25-30 min of reaction. The appearance of violet color indicates the gradual evolution of NPs in the solution. Curve f, Fig. 1A shows the SPR band of Os particles peaking above $500 \mathrm{~nm}$ although due to a smaller absorbance value, the SPR peak is not clearly visible in Fig. 1A. The inset of Fig. 1A shows a camera image of the dark violet Os NPs solution. Fig. 1B shows enlarged UV-Vis spectra for the appearance of a SPR band of Os particles, as seen in Table 1. Curve a, Fig. 1B shows a sharp absorption band at $530 \mathrm{~nm}$ due to the SPR of small size Os nano spheres. Curve b, Fig. 1B shows a broad absorption band with two small humps at 488 and $551 \mathrm{~nm}$ due to the presence of
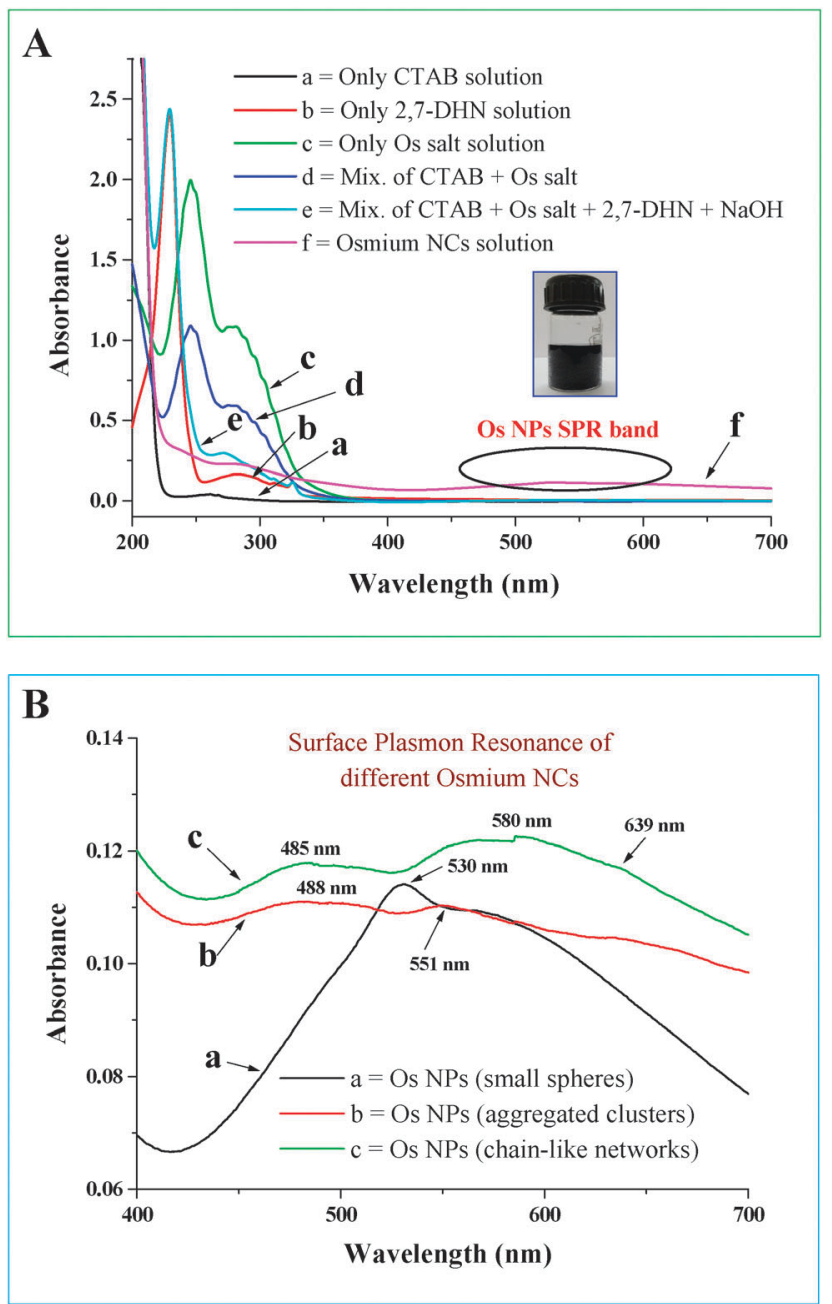

Fig. 1 (A) UV-Vis absorption spectra of Os NCs at different stages of the synthesis process. Curve (a) is the absorption band of an aqueous CTAB solution; (b) absorption band of 2,7-DHN solution; (c) absorption band of aqueous $\mathrm{OSO}_{4}$ salt solution; (d) absorption band of a mixture of CTAB with Os salts; (e) absorption band for the mixture of alkaline 2,7-DHN with CTAB and Os salt; (f) SPR band of Os particles. The inset shows a camera image of the dark violet Os NPs solution. (B) Enlarged UV-Vis spectra for the appearance of a SPR band of Os particles with different morphologies. Curves (a), (b) and (c) are the SPR band for Os particles having different morphologies like small size spheres, aggregated clusters and chain-like network structures, respectively.

aggregated clusters in the sample. Curve c, Fig. 1B shows three distinct absorption bands at 485, 580 and $639 \mathrm{~nm}$ due to the SPR of Os NPs having chain-like networks. Other than a couple of recent papers, ${ }^{21}$ the experimental absorption spectrum of 
Os NPs has not been reported. The Os nanochain SPR band reported $^{21}$ single band maxima at $\sim 540 \mathrm{~nm}$ although in our case we observed the shifting of peak positions at a higher wavelength due to aggregation of individual particles forming NCs and in-turn the NCs aggregating to form a chain-like network structure. We recently reported that self-assembled Os NCs in DNA having a wire-like morphology can generate a SPR peak at $594 \mathrm{~nm}$ whereas a honey-comb like structure can generate a peak at $602 \mathrm{~nm} .^{22}$ In our present study, the appearance of a peak at a much higher wavelength region (at $639 \mathrm{~nm}$ ) is probably attributed to plasmon oscillation originating for the formation of coupled NPs as observed in the TEM microscopy images. Similar types of explanation were drawn by Sampath and co-workers as they also observed a peak at $540 \mathrm{~nm}$ for their Os nano-chain aggregates. ${ }^{21}$ Creighton et al. reported earlier a theoretical calculation that Os NPs with a spherical shape can show a SPR band near $350 \mathrm{~nm}$ with large absorption below $300 \mathrm{~nm} .{ }^{32}$ Although it has already been established with other metal NPs that the SPR band can be shifted towards a higher wavelength region while increasing the particle size due to aggregation or self-assembly of individual particles. It is also reported that aggregation of metal NPs like Au NPs leads to a red shift in the plasmon band due to the electric dipole-dipole interaction leading to coupling between the plasmon oscillations of different particles. ${ }^{33}$ They observed that the color of Au NP solution turns from red to blue/purple due to the red shift in the plasmon band towards a longer wavelength i.e. red region of the electromagnetic spectrum.

\section{Transmission electron microscopy (TEM) analysis}

Fig. 2 shows transmission electron microscopy (TEM) images of the Os NPs with different morphologies synthesized using the described procedure given in the Experimental section and in Table 1. Fig. 2A and B show a TEM image of the aggregated NCs at low and high magnifications. From the image we can see that the smaller particles are aggregated together to form clusters. The average diameter of the small Os particles is $\sim 2 \pm 0.2 \mathrm{~nm}$, they are aggregated together to form the NCs. The average diameter and lengths of the aggregated clusters are $\sim 70 \pm$ $10 \mathrm{~nm}$ and $250 \pm 20 \mathrm{~nm}$, respectively which consist of a huge number of smaller particles. The inset of Fig. $2 \mathrm{~B}$ shows the corresponding selected area electron diffraction (SAED) pattern which confirms the particles are non-crystalline in nature. Fig. 2C and D shows a TEM image at low magnification of the Os nano chain-like networks. Fig. 2E and F shows a TEM image of the chain-like networks at higher magnification. From the image it is clear that smaller Os particles are self-assembled together and generate a chain-like network structure. The high magnified image in Fig. 2F shows a single chain network. The average diameter of the nano chain-like network is $\sim 10 \pm 2 \mathrm{~nm}$ while the approximate length of the networks is $\sim 300 \pm 50 \mu \mathrm{m}$. Careful observation shows that a network structure is generated due to assemblies of small spherical particles with a diameter $\sim 1.5 \pm 0.5 \mathrm{~nm}$. The inset of Fig. 2F shows the corresponding SAED pattern which states that the particles are non-crystalline in nature. Fig. 2G and $\mathrm{H}$ shows a low and high magnified TEM
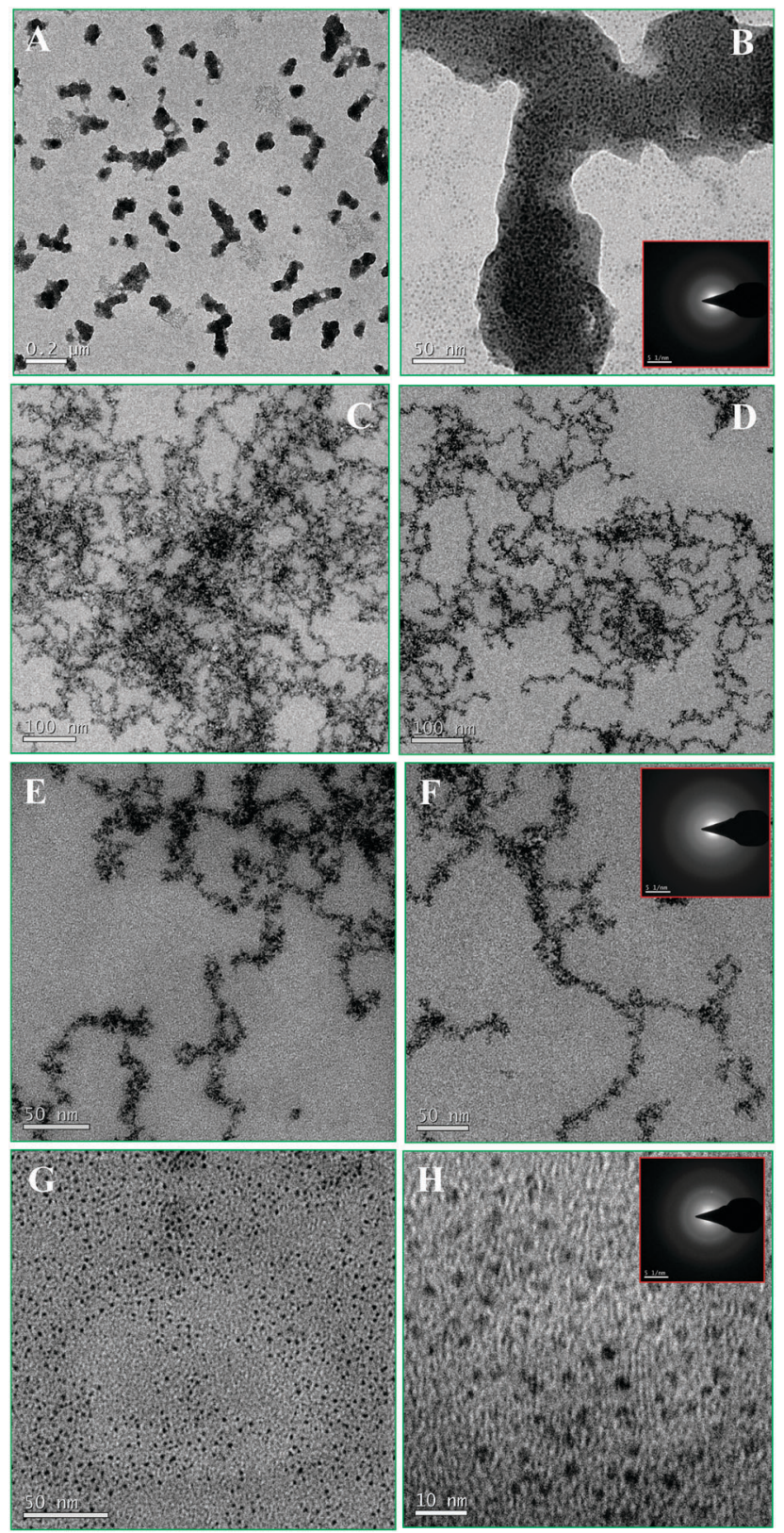

Fig. 2 Transmission electron microscopy (TEM) images of the Os NCs with different morphologies synthesized in different reaction conditions. (A) and (B) show a low and high magnified TEM image of the aggregated NCs. The inset of (B) shows the selected area electron diffraction (SAED) pattern. (C) and (D) show a low magnified TEM image of the Os chain-like networks. (E) and (F) show a high magnified TEM image of the Os chain-like networks. The inset of Fig. 2F shows the corresponding SAED pattern. (G) and (H) show the low and high magnified TEM image of Os NPs with spherical shapes and the inset of $(H)$ shows the corresponding SAED pattern.

image of Os NPs having spherical shapes. From the image it can be seen that the average diameter of the spheres is $\sim 2 \pm 0.5 \mathrm{~nm}$ and the particles are nicely dispersed and form uniform size particles. The inset of Fig. $2 \mathrm{H}$ shows the corresponding SAED pattern which indicates the non-crystallinity of the particles. In the case of non-crystalline materials, the feature of concentric rings in SAED disappears but only leaves 
a hallow around the bright spot in the center, which indicates that the electrons are diffracted randomly by a material of amorphous structure. So from the TEM analysis, we confirmed that Os NPs with different morphologies can be synthesized by changing the reaction parameters as given in the Experimental section under Table 1.

\section{Energy dispersive X-ray spectroscopy (EDS) and X-ray diffraction (XRD) analysis}

Fig. S1 (in the ESI $\dagger$ ) shows energy dispersive X-ray spectroscopic (EDS) analysis of the synthesized Os NPs in CTAB surfactant media. The EDS spectrum consists of the expected peaks for $\mathrm{Ca}$, $\mathrm{Si}, \mathrm{Na}, \mathrm{O}$, Os and $\mathrm{Br}$. The details of the EDS analysis are given in the ESI. $\dagger$ Fig. S2 (in the ESI $\dagger$ ) shows an X-ray diffraction pattern of the CTAB stabilized Os NCs. We analyzed the XRD of all three morphologies although here we are showing the XRD of the chain-like Os particles. The diffraction observed from the (002), (101), (102), (110) and (103) planes of the hexagonal phases of osmium which matches nicely the JCPDS card no: 06-0662. ${ }^{20,21,34}$ As we observed from Fig. S2 (ESI $\dagger$ ), there is no sharp or high intense peak is observed even by slow scanning during the XRD data collection, probably due to the very small size of the synthesized CTAB-Os particles. This also was further proven by the SAED pattern during TEM analysis, as discussed above. Sharp XRD peaks at high angles are not observed, which indicates larger Os particles are not formed in our process. A broad peak at a lower angle near $30^{\circ}$ is observed, which is probably due to the crystallization of the CTAB molecules which is used as a stabilizing agent during our synthesis process. The intensities of the peaks are low and the broad crystallographic peaks at low angular values signify the small size of the particles as observed by Borja-Arco et al. ${ }^{18}$ and Sampath et al. ${ }^{21}$ earlier.

\section{Fourier transform infrared spectroscopy (FT-IR) analysis}

The FT-IR spectra of only CTAB and CTAB bound Os NCs are shown in Fig. 3. We plot the FT-IR spectra of only CTAB with

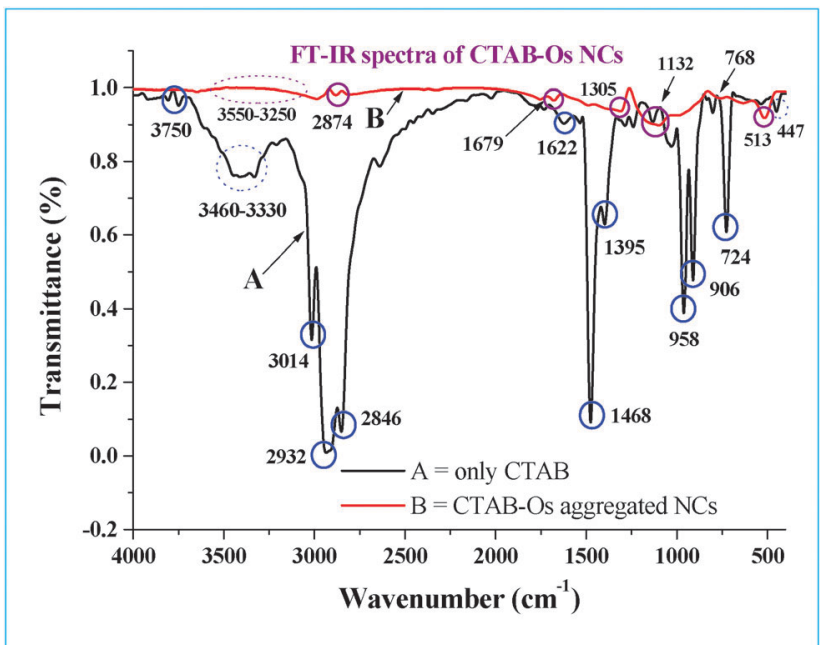

Fig. 3 FT-IR spectra of pure CTAB (curve A) and CTAB bound Os NCs (curve B).
CTAB bound Os NCs which supports the capping action of CTAB with the Os NCs surface and also provides an idea of the types of interaction taking place at the NCs surface. As an example, we have plotted the FT-IR spectra of CTAB-Os having an aggregated cluster morphology although all other morphologies also giving similar types of the FT-IR pattern which is quite expected as all the case particles stabilized the CTAB molecule. Fig. 3, curves A and B denote the FT-IR spectra of pure CTAB and CTAB bound Os NCs, respectively. Due to the low transmittance (\%) value of CTAB-Os NCs as compared to only CTAB, the intensity of the peaks for CTAB-Os NCs is low while plotting both together. An enlarged view of the FT-IR spectra of CTAB-Os NCs is shown in the ESI $\dagger$ as Fig. S3. It is important to mention that the FT-IR spectra of CTAB capped metal or metal NPs strongly depend upon the particle size and shape. ${ }^{35}$ The $\mathrm{C}-\mathrm{H}$ stretching and anti-stretching vibration frequencies of pure CTAB are observed at 2846 and $2932 \mathrm{~cm}^{-1}$ while in the case of CTAB bound Os NCs, only a single band was observed at $2874 \mathrm{~cm}^{-1}$ which indicates the presence of $-\mathrm{CH}_{2}$ vibrational frequencies on the Os NCs surface which in-turn indicates the capping action of CTAB on the NCs surface. A broad band at $3330-3460 \mathrm{~cm}^{-1}$ for pure CTAB corresponds to the $\mathrm{N}-\mathrm{H}$ stretching vibration for pure CTAB. Now for CTAB-Os NCs, the broad band is broader and appears at around $\sim 3250-3550 \mathrm{~cm}^{-1}$ which indicates an interaction of CTAB with the Os NCs surface. CTAB has a positively charged ammonium group on its polar head side which binds with the Os NCs surface via Os-N bond formation. For pure CTAB, a strong but less intense peak is observed at $3014 \mathrm{~cm}^{-1}$ which shifts for the CTAB-Os NCs sample. This peak arises due to the presence of a secondary amine group and these results indicate that the CTAB head group faces the Os NCs surface. A sharp but low intense peak at $\sim 3750 \mathrm{~cm}^{-1}$ in pure CTAB is due to the $\mathrm{O}-\mathrm{H}$ stretching frequency of water solvating the surfactant CTAB. The $\mathrm{O}-\mathrm{H}$ vibrational peak is absent in a CTAB bound Os NCs sample which indicates the absence of any water molecule or moisture in the Os NCs sample. The region $1300-1680 \mathrm{~cm}^{-1}$ is signifying due to $\mathrm{CH}_{2}$ scissoring mode of vibration and the $\mathrm{O}-\mathrm{H}$ bending mode of water around the bound head group. All these sharp peaks in pure CTAB are also present or slightly shifted but with low intensity in the case of CTAB bound Os NCs which indicates an interaction of CTAB with the Os NCs surface. The strong peak at 724,906 , and $958 \mathrm{~cm}^{-1}$ for pure CTAB is either broadened or shifted in the case of CTAB bound Os NCs which indicates an interaction of CTAB with Os NCs. Most of the peaks described above bear similarity to earlier reports for CTAB capped metal nanostructures. ${ }^{3,36}$ So from the above FT-IR analysis, it is clear that Os NCs are stabilized by the CTAB molecule probably via the Os- $\mathrm{N}$ bond formation due to the presence of an ammonium ion on its surface by forming a double layer arrangement.

\section{Reaction mechanism for the formation of Os nanostructures of different morphologies}

We have conducted a few control experiments to figure out the possible reaction mechanisms and the corresponding discussions were given in the ESI. $\dagger$ Os nanostructures with different morphologies can be synthesized by reduction of Os salt with alkaline 2,7-DHN in CTAB micellar media. In the absence of 
any of these reagents like Os salt or 2,7-DHN or NaOH, Os NPs were not formed in the proposed reaction conditions. We have measured the $\mathrm{pH}$ of the different solution and solution mixtures during the synthesis process. The $\mathrm{pH}$ of only CTAB solution $\left(\sim 10^{-2} \mathrm{M}\right)$ is 7.72 , that of Os salt $\left(\sim 10^{-2} \mathrm{M}\right)$ is 5.89 . The $\mathrm{pH}$ of a mixture of CTAB, Os salt and 2,7-DHN is 6.66 and the mixture of all the four reagents including $\mathrm{NaOH}$ is 6.43 (before particles formation). The $\mathrm{pH}$ of the Os NPs solution is 7.13 for aggregated cluster, 7.72 for chain-like networks and 6.88 for nano spheres. Fig. S4A (in the ESI $\dagger$ ) shows a TEM image of aggregated Os NPs while $\mathrm{NaOH}$ concentration is very high and Fig. S4B (in the ESI $\dagger$ ) shows an image of a nanocube while the $\mathrm{NaOH}$ concentration is low. The inset of Fig. S4B (ESI $\dagger$ ) shows a SAED pattern which signifies a perfectly single crystalline nature of the cubes which we did not observe from other Os nanostructures, probably due to the smaller size of the particles. The Os nanostructures having different morphologies can be synthesized by reduction of Os salt with alkaline 2,7-DHN in CTAB micellar media. In the absence of any of these reagents like Os salt or 2,7-DHN or $\mathrm{NaOH}$, Os NPs are not formed in the proposed reaction conditions. We have measured the $\mathrm{pH}$ of the different solution and solution mixtures during the synthesis process. The $\mathrm{pH}$ of only the CTAB solution $\left(\sim 10^{-2} \mathrm{M}\right)$ is 7.72 , the Os salt $\left(\sim 10^{-2} \mathrm{M}\right)$ is 5.89. The $\mathrm{pH}$ of a mixture of CTAB, Os salt and 2,7-DHN is 6.66 and the mixture of all the four reagents including $\mathrm{NaOH}$ is 6.43 (before particles formation). The $\mathrm{pH}$ of the Os NPs solution is 7.13 for an aggregated cluster, 7.72 for chain-like networks and 6.88 for nano spheres. The formation mechanism of Os particles seems to proceed via several steps. Initially, after addition of $\mathrm{NaOH}$ with CTAB, 2,7-DHN and Os salt, there is formation of a perosmate anion having the formula $\left[\mathrm{OsO}_{4}(\mathrm{OH})_{2}\right]^{2-}$. The perosmate anion is in alkaline solution easily reduced to osmate anion having a formula $\left[\mathrm{OsO}_{2}(\mathrm{OH})_{4}\right]^{4-}$. Now, this osmate anion is reduced by alkaline 2,7-DHN and generate the small Os(0) nuclei. The reducing capability of the hydroxylic or phenolic compounds for the generation of NPs has already been reported. ${ }^{33-37}$ It has been reported that different compounds like TX-100, ${ }^{37}$ ascorbic acid, ${ }^{38}$ benzophenone, ${ }^{39}$ polyvinyl alcohol, ${ }^{40}$ dendrimer $^{41}$ can reduce metal ions to metal NPs either in the presence of $\mathrm{NaOH}$ or passing UV light or microwave heating. Kundu et al. reported earlier that different beta-diketones having similar types of functional group can reduce $\mathrm{Au}(\mathrm{III})$ ions to $\mathrm{Au}(0) .{ }^{42}$ Moreover, very recently, it was also reported that deoxyribonucleic acid (DNA), having a hydroxyl group on its sugar part can also reduce metal ions to generate metal NPs. ${ }^{43,44}$ So, we believe that by the reaction of alkali and 2,7-DHN, an anionic species or radical anions or solvated electrons are generated which is responsible for the reduction of Os ions to Os(0). Initially, the reduction of Os ions to $\operatorname{Os}(0)$ leads to the formation of small spherical nuclei. The nuclei subsequently formed Os atoms and assembled them together into a crystalline particle. At this stage, the surfactant $\mathrm{CTAB}$ can play a major role for the formation of a different morphology of Os nanostructures. The CTAB molecule can attach the surface of these crystals and slow down the growth rate of different crystal facets. When the surfactant concentration is less $\left(10^{-5} \mathrm{M}\right.$, same as stock solution), the interaction of the cationic part of the surfactant with the specific surfaces of the crystalline particle is weak. As a result, the growth of the Os particles takes place in all possible directions and generates spherical particles as shown in Scheme 1. When the surfactant concentration is moderately high $\left(\geq 10^{-4} \mathrm{M}\right)$, CTAB itself can form a worm-like or rod-like micellar template, as reported by others ${ }^{45,46}$ and the crystalline particles can grow on that specific template and generates a chain-like morphology. For the formation of wire-like or rod-like gold NPs were synthesized earlier utilizing a similar concept in the CTAB surfactant media. ${ }^{45}$ Those chain-like structures were formed in the solution and with increase in the reaction time can further self-assemble together to generate a network-like structure, as shown in the TEM images of Fig. 2C-F and also in Scheme 1. While the CTAB concentration we used was much higher $\left(\sim 10^{-2}-10^{-3} \mathrm{M}\right)$, the crystalline small particles which were initially formed are aggregated together to form a cluster-like morphology. The formation mechanism of the three different morphologies is schematically shown in Scheme 1. From Scheme 1 , it is clear that at low concentration only spherical particles are formed, at medium concentration chain-like networks were formed and at moderately high concentration the aggregated clusters were formed. The formation of nano-chains by the reduction of Os salt with ascorbic acid at high temperature ( $\sim 95{ }^{\circ} \mathrm{C}$ ) has been reported..$^{21}$ The authors observed that initially smaller size Os nuclei are formed and they react with each other to generate small Os NPs, and finally these small particles can reach an equilibrium size and further self-assemble to form nanochains. ${ }^{21}$ In the present study, we also believed that initially small spherical nuclei were formed and finally depending upon the concentration of the stabilizing agent the particles grow in different manner and generate a specific morphology, as shown in Scheme 1. Although, at this point, we were not fully convinced about the generation of a different morphology, further analysis and more control experiments were necessary to get an in-depth idea of the growth process. After the generation of different morphologies of Os nanostructures, we used them for possible application purposes in catalysis for the reduction of aromatic nitro compounds and as a potential substrate in a SERS study which is shown schematically in the ESI $\dagger$ as Scheme S1.

\section{Catalysis study on the reduction of 4-NA using Os NCs}

An in-depth study was undertaken on $\mathrm{NaBH}_{4}$ reduction of 4-NA in the presence of CTAB stabilized Os NPs having different morphologies as catalysts. As an example, a detailed study was performed with 4-NA as model examples although several other nitro compounds were also tested and that is discussed in a later part of this section. The detailed control experiment for the catalysis study and the corresponding UV-Vis (Fig. S5, ESI $\dagger$ ) spectra were discussed in the ESI. $\dagger$ For a typical catalysis study, we mixed $4 \mathrm{~mL}$ of DI water with $500 \mu \mathrm{L}$ of $10^{-3} \mathrm{M} 4$-NA solution. The solution became light yellowish in color. Now, $1 \mathrm{~mL}$ of $10^{-2}$ M freshly prepared ice-cold $\mathrm{NaBH}_{4}$ was added, mixed well and finally $400 \mu \mathrm{L}$ of Os NPs solution (aggregated cluster-like morphology) was added. It is important to mention here that the whole catalysis reaction was done with Os aggregated cluster-like morphology although other morphologies were also 


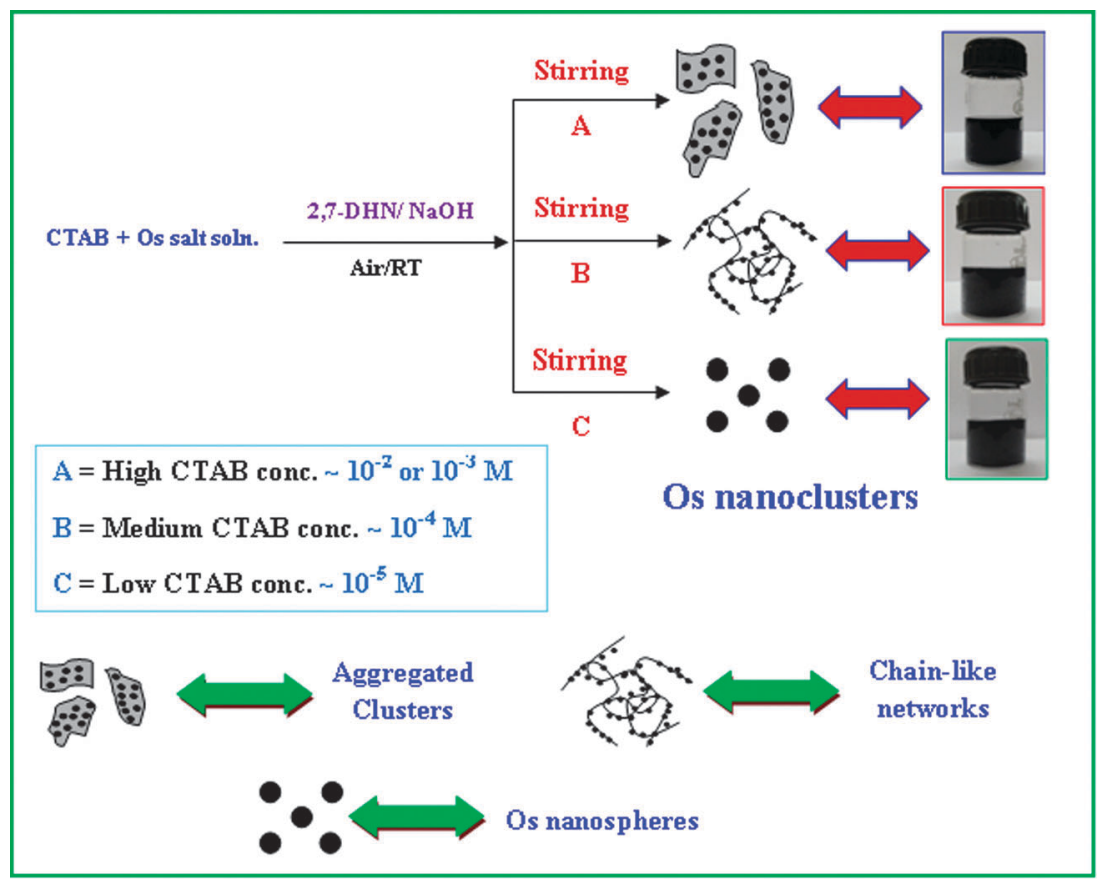

Scheme 1 Formation mechanism of three different morphologies of osmium NPs in CTAB.

tested as a preliminary study. The $\mathrm{pH}$ values of the different solution mixtures are given in the ESI. $\dagger$ The UV-Vis absorption spectrum of 4-NA with $\mathrm{NaBH}_{4}$ solution appeared at $381 \mathrm{~nm}$ due to intermolecular charge transfer spectra. ${ }^{47}$ The progress of the reduction can be well visualized by the disappearance of a $381 \mathrm{~nm}$ peak and the appearance of a new peak at $239 \mathrm{~nm}$ due to the formation of the reduced product para-phenylenediamine ( $p$-PDA) in the solution. The $381 \mathrm{~nm}$ peak decreases gradually and the $239 \mathrm{~nm}$ peak increases gradually with time, as seen in Fig. 4A. From Fig. 4A, it was seen that the reaction was completed within 10 min using Os aggregated cluster-like morphology as a catalyst which can be confirmed by a change in the solution color (yellowish to colorless) and from the absorbance value in the UV-Vis spectrum. The inset of Fig. 4A shows the yellowish color of 4-NA solution (before reduction) and the colorless $p$-PDA solution after reduction. In the reaction we have used a large excess of $\mathrm{NaBH}_{4}$ concentration so that its concentration became constant throughout the reaction. Moreover, due to the presence of excess $\mathrm{NaBH}_{4}$, the liberated $\mathrm{H}_{2}$ gas can prevent aerial oxidation of the product. As the $\mathrm{BH}_{4}{ }^{-}$concentration remains constant throughout the reaction, the catalysis reaction of 4-NA is considered to follow the pseudo-first order kinetics with respect to 4-NA. Fig. 4B shows the $\ln (\mathrm{Abs}) v s$. time $(T, \mathrm{~min})$ plot for the reduction of 4 -NA. The rate constant value obtained from the reaction is $0.26 \mathrm{~min}^{-1}$ and the correlation coefficient and standard deviation values were 0.982 and 0.185 , respectively. The pseudo-first order rate equation is $\ln [A]=\ln \left[A_{0}\right]+0.98 \times t$ where $[A]$ is the absorbance value at a time $t,\left[A_{0}\right]$ is the initial absorbance value and $t$ is the reaction time. The formation of the final product $p$-PDA is confirmed from the UV-Vis spectra as well as from the ${ }^{1} \mathrm{H}-\mathrm{NMR}$ spectra (not shown here). The catalytic efficiency of the Os aggregated clusters as a catalyst has been compared with other literature reports where it was found that our catalyst was superior to any other reported methods. ${ }^{43,44,48-51}$ It is important to note that except for our recent report, ${ }^{21}$ there was no example available in the literature where Os NPs were used for the catalysis reaction of the organic nitro compounds, so we compared our catalytic efficiency with other noble metals like $\mathrm{Au}$ and Ag. ${ }^{43,44,48-51}$ The anisotropic Au NPs also used as a catalyst for the 4-NA reduction and the rate constant value were found to be $2.083 \times 10^{-2} \mathrm{~min}^{-1}$. In our previous report taking Os NCs in DNA as scaffold, we observed the first order catalytic reaction rate $3.87 \times 10^{-2} \mathrm{~min}^{-1}$ for first $6 \mathrm{~min}$ and $0.45 \mathrm{~min}^{-1}$ for later $8 \mathrm{~min} .^{22}$ In the present study we observed that the reaction rate was $0.26 \mathrm{~min}^{-1}$ which was the highest ever reported for Os NPs as a catalyst and much superior to other noble metals like $\mathrm{Au}$ or Ag. ${ }^{2,22,43,44,48-51}$ Apart from 4-NA, we also checked our catalysis reaction with other nitro compounds like 4-NP, 2-NP, 2-NA, etc., and in all cases the expected catalysis reduction taking place and the reaction were completed in different time scales. Table S1 (in the $\mathrm{ESI} \dagger$ ) shows the different nitro compounds tested and the corresponding time required for the full reduction. As a preliminary experiment, we also tested the role of the different morphologies on the catalysis reaction. We have seen that keeping all reaction parameters fixed, the reaction was the fastest with an Os aggregated cluster-like morphology, a medium with nano spheres, and the slowest with a chain-like network. So the order of the catalysis reaction rate follows as: aggregated cluster $>$ nano-spheres $>$ chainlike network. Scheme S2 (in the ESI $\dagger$ ) shows different catalysis rates observed for different morphologies of Os nanostructures. It is well known that in a homogeneous catalysis reaction, the catalytic rate depends mainly on two important parameters. One is the effective surface area available and the other is the availability of catalyst particles in the reaction medium. The higher catalytic rate for aggregated clusters might be due to their overall increase in size due to aggregation. Careful observation says that the individual 

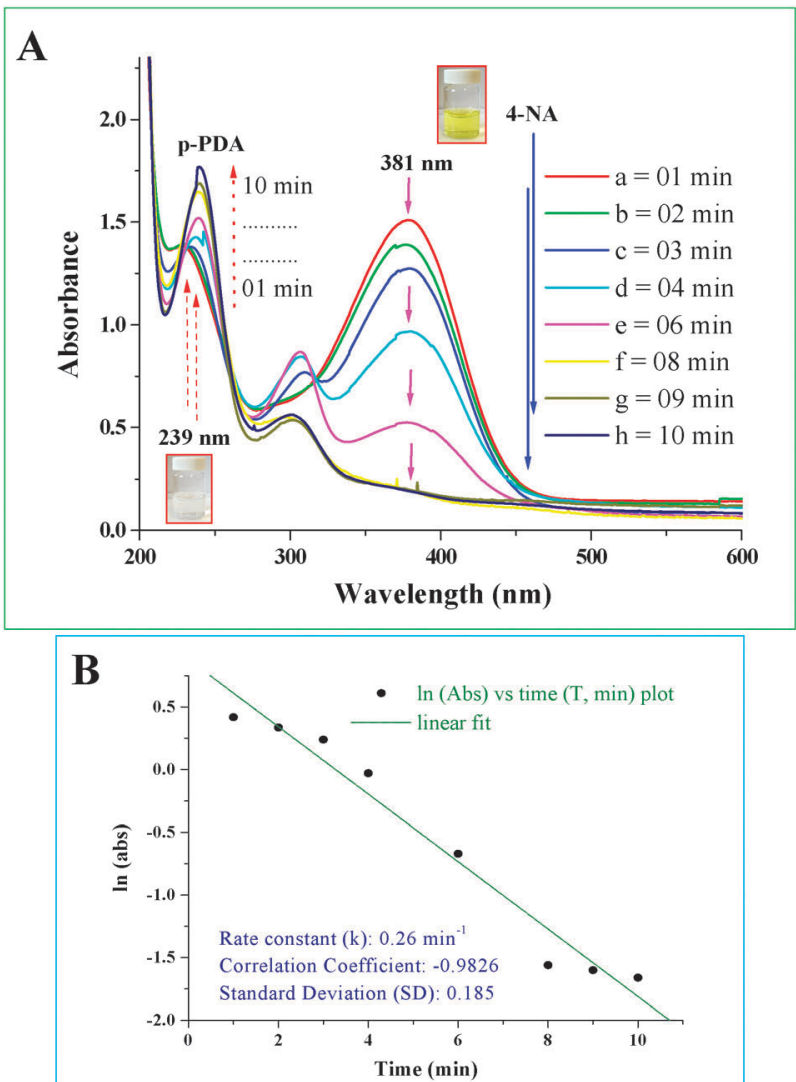

Fig. 4 (A) Successive decrease of the UV-Vis absorption spectrum for reduction of 4-NA with Os aggregated clusters as a catalyst in the presence of $\mathrm{NaBH}_{4}$. The inset shows the yellowish color of 4-NA solution (before reduction) and the colorless $P$-PDA solution after reduction. (B) Shows the In (Abs) vs. time ( $T, \mathrm{~min}$ ) plot for the reduction of 4-NA having a rate constant value of $0.26 \mathrm{~min}^{-1}$.

spherical Os particles having a diameter between 1-3 nm are assembled together and generate a large aggregated cluster where the effective surface area is increased as compared to individual particles. Due to the increase in effective surface area, the electron transfer from the reducing agent (here it is $\mathrm{NaBH}_{4}$ ) to the nitro compound becomes more feasible and faster which in turn increases the catalytic rate tremendously. Apart from this, for a certain volume, the number of particles should be more in case of aggregated clusters as compared to other morphologies, which also facilitates the higher catalytic rate for aggregated clusters, although the exact reason for the different catalytic rates of various morphologies was not clear and further study is needed for an in-depth idea on this.

\section{Surface enhanced Raman scattering (SERS) study using CTAB stabilized Os NPs having different morphologies}

Since the discovery of SERS by Fleischmannn et al. in $1974,{ }^{52}$ exhaustive efforts by a number of researchers have been devoted to the development of a practical substrate to investigate and utilize the SERS effect. The mechanism and theory of SERS are still under a considerable debate. In general, there are two possible mechanisms described in the literature: one is the electromagnetic (EM) effect and the other is the chemical effect. It is assumed that the oscillating electric field of a light beam induces an oscillating dipole in a colloidal metal particle. If the natural frequency of oscillation of the conduction band electron matches with the frequency of light, the plasmon resonance takes place and the induced dipole becomes large.

In the case of metal NPs, the EM effect is predominant which implies that the excitation energy was not distributed uniformly over the particles but was localized in some specific places called 'hot spots'. It has already been discussed in an earlier report that the size and shape of the NPs strongly affect the SERS phenomenon. Creighton et al. described the SERS effect on the spherical aggregates of $\mathrm{Ag} \mathrm{NPs}{ }^{53}$ It was also reported that aggregation of NPs can generate better enhancement as compared to monodispersed spherical particles. ${ }^{54-56}$ Recently, the effect of the NPs shape was also tested in a SERS study and it was observed that anisotropic NPs like nanoprisms and nanorods give a better SERS signal as compared to spherical particles. ${ }^{5}$ Recently, Kundu et al. studied that aggregated Ag NCs can generate better SERS as compared to a monomer or a dimer and the results were compared to theoretical calculations. ${ }^{57}$ Most of the previous studies on SERS however, mainly focus on a few noble metal NPs like $\mathrm{Au}, \mathrm{Ag}, \mathrm{Cu}, \mathrm{Pd}, \mathrm{Pt}$, etc., and a vast study is centered on $\mathrm{Au}$ and $\mathrm{Ag}$ only. Very recently, Os nanostructures were also tested in SERS studies. ${ }^{21,22}$ As we observed, the plasmon resonance of our synthesized Os

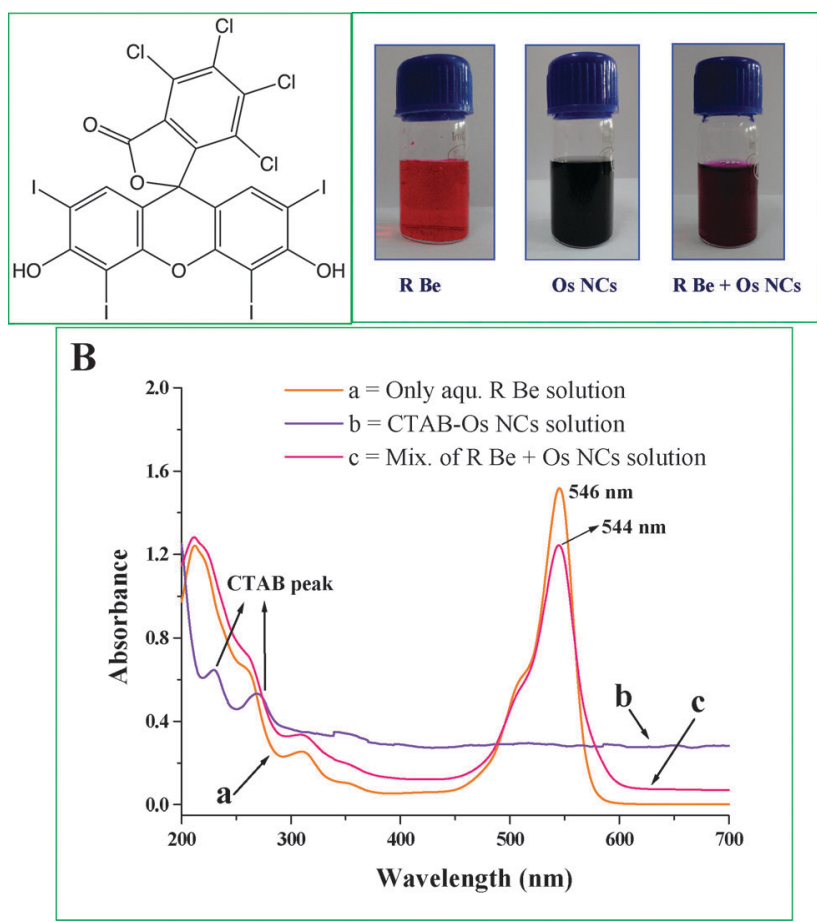

Fig. 5 (A) Chemical structure of $\mathrm{R}$ Be on the left and right side contains a three camera image with only R Be solution (left), Os NCs solution (middle) and $\mathrm{R} \mathrm{Be}-\mathrm{Os} \mathrm{NCs}$ solution mixture (extreme right). (B) UV-Vis spectra of different solutions mixture before the SERS experiment; curve (a) is the absorption spectrum of only aqueous R Be solution; (b) is the SPR band of CTAB-Os NCs solution; (c) is the absorption band of the mixture of R Be and Os NCs solution. 


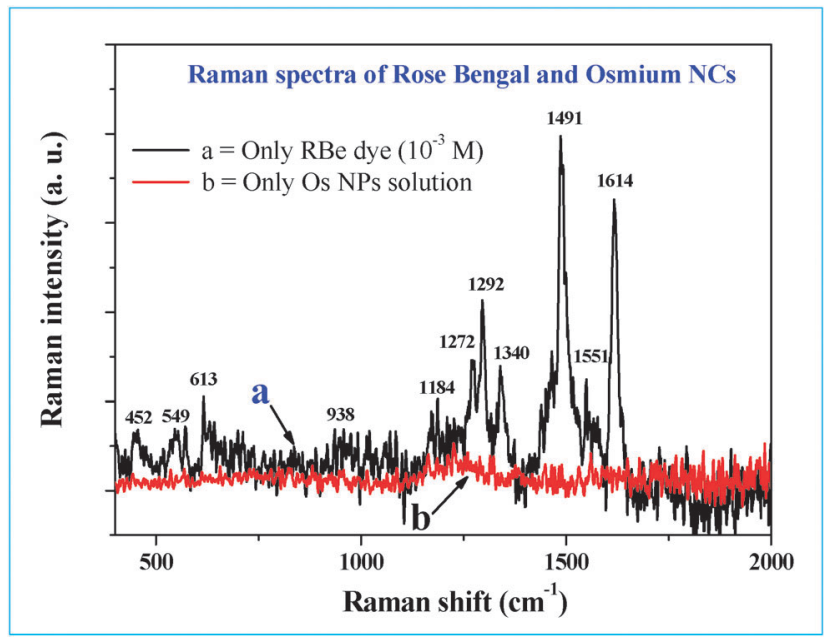

Fig. 6 Normal Raman spectra of R Be dye at $10^{-3} \mathrm{M}$ (curve a) and only aqueous Os NCs solution (curve b).

particles falls in the visible region, so we believed that Os NPs can also act as a potential SERS substrate. Keeping that idea in mind, we performed a SERS study of Os NPs taking Rose Bengal ( $\mathrm{R} \mathrm{Be}$ ) as a model anionic dye molecule. We chose $\mathrm{R}$ Be for better adsorption due to the opposite charge to the CTAB-Os particles.

As we observed, the highest enhancement includes Os NPs with aggregated cluster like morphology, a detailed study was performed with that morphology although other morphologies were also examined for comparison purposes. The details about the concentration of the reagents used for the SERS study is given in the Experimental section. A stock solution of $10^{-3} \mathrm{M} \mathrm{R}$ Be dye solution was used and the stock solution was diluted and a different solution of lower concentration was prepared. Fig. 5A shows the chemical structure of $\mathrm{R} B$ at the left and right side containing three camera images with only $\mathrm{R} B$ solution (left), Os NCs solution (middle) and R Be-Os NCs solution mixture (extreme right). Fig. 5B shows the UV-Vis spectra of different solution mixtures before performing the SERS experiment. Curve a, Fig. 5B shows the absorption spectra of only aqueous $\mathrm{R}$ Be solution which has a high intensity band at $546 \mathrm{~nm}$ and low intensity bands at $310 \mathrm{~nm}$ and $261 \mathrm{~nm}$. Curve b, Fig. 5B shows the SPR band of CTAB-Os NCs solution.

Table 2 Position of the Raman bands and assignments of the peaks for $\mathrm{R} B e$

\begin{tabular}{|c|c|c|c|}
\hline $\begin{array}{l}\text { Raman bands for } \\
\text { R Be (reported) }\end{array}$ & $\begin{array}{l}\text { Raman bands for } \mathrm{R} \text { Be } \\
\text { in water (observed) }\end{array}$ & $\begin{array}{l}\text { R Be-Os NCs } \\
\text { (SERS) (observed) }\end{array}$ & Assignment of peaks \\
\hline 616 & 613 & 613 & $\nu(\mathrm{C}-\mathrm{I})+\delta(\mathrm{CCO})$ \\
\hline 761 & - & 763 & $\nu(\mathrm{C}-\mathrm{Cl})$ \\
\hline 958 & 938 & 949 & \\
\hline 1012 & - & 1011 & $\nu(\mathrm{C}-\mathrm{OH})$ \\
\hline 1166 & 1184 & 1173 & $\nu(\mathrm{C}-\mathrm{O})+\nu(\mathrm{C}-\mathrm{C})+\delta(\mathrm{C}-\mathrm{H})$ \\
\hline 1270 & 1272 & 1271 & $\delta(\mathrm{CCC}) \operatorname{ring}+\delta(\mathrm{C}-\mathrm{H})$ \\
\hline 1297 & 1292 & 1292 & $\delta(\mathrm{CCC}) \operatorname{ring}+\delta(\mathrm{C}-\mathrm{H})$ \\
\hline 1340 & 1340 & 1344 & $\nu(\mathrm{C}-\mathrm{C}) \mathrm{ring}$ \\
\hline 1491 & 1491 & 1491 & $\nu_{\text {as }}(\mathrm{C}=\mathrm{C})$ ring \\
\hline 1553 & 1551 & 1549 & $\nu(\mathrm{C}-\mathrm{C})$ ring \\
\hline 1615 & 1614 & 1614 & $\nu_{\mathrm{s}}(\mathrm{C}=\mathrm{C})$ ring \\
\hline
\end{tabular}

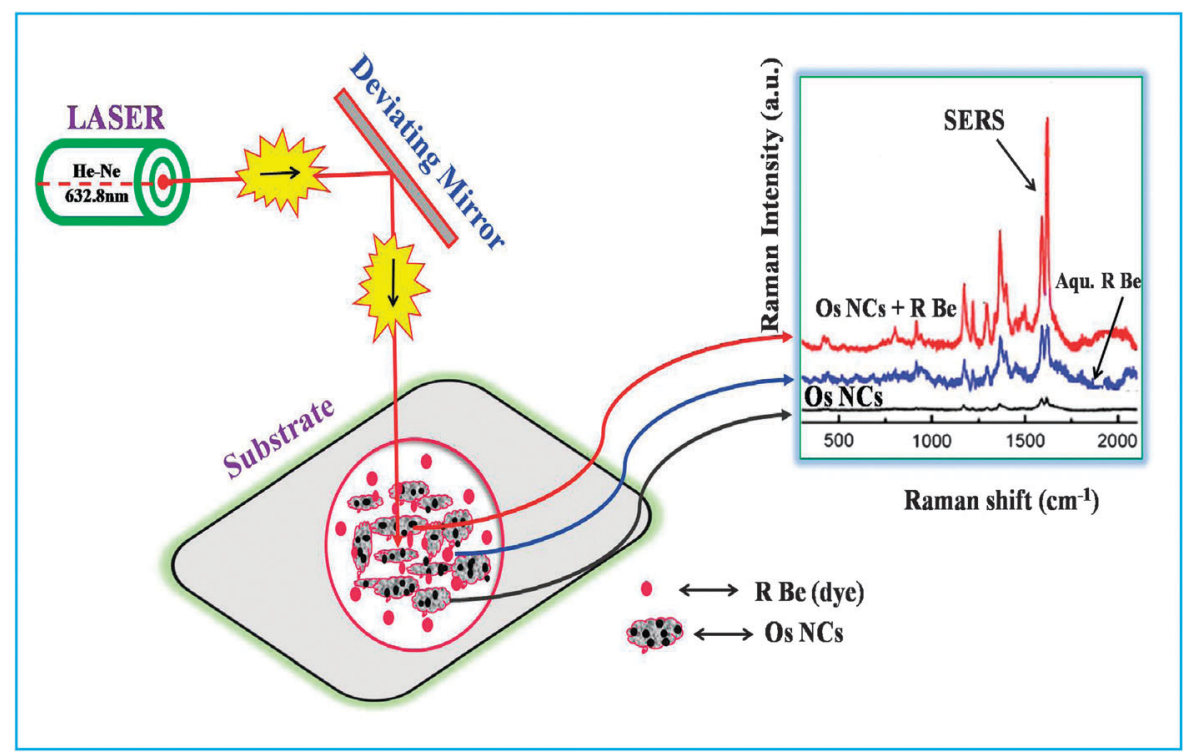

Scheme 2 Schematic representation of a SERS study considering Os NCs and R Be dye molecule. 
Curve c, Fig. 5B shows the absorption band of a mixture of $\mathrm{R} B e$ and Os NCs solutions. There is a small shift of the absorption maxima of $\mathrm{R}$ Be taking place, which indicates the absorption or interaction of the dye with the NPs surface. It is important to note that $\mathrm{R}$ Be is a typical anionic dye which adsorbs with the Os NCs surface via physical adsorption or via electrostatic interaction due to the opposite charge of the CTAB capped Os NCs. Fig. 6 shows normal Raman spectra of a R Be dye $\left(10^{-3} \mathrm{M}\right)$ and only aqueous Os NCs solution, as indicated by curve a and curve $b$, respectively. There is no significant band observed for the Os NCs sample. The position of the Raman bands and the assignments of the peaks for $\mathrm{R}$ Be are summarized in Table 2 . The assignments are performed on the basis of published data on $\mathrm{R}$ Be and related xanthene molecules. ${ }^{58}$ From Table 2, it can be seen that there is shifting of the spectral peak positions in a few cases for $\mathrm{R}$ Be which mixes with CTAB-Os NCs (SERS spectra) as compared to only R Be. Scheme 2 shows a schematic representation of the SERS study concerning Os NCs and a $\mathrm{R}$ Be dye molecule. Fig. 7A shows SERS spectra for different concentrations of the $\mathrm{R}$ Be molecule. Curve a, Fig. 7A shows Raman spectra of only a $\mathrm{R}$ Be dye at $10^{-3} \mathrm{M}$ concentration and curve $\mathrm{b}-\mathrm{f}$ shows SERS spectra at a dye concentration of $10^{-3} \mathrm{M}, 10^{-4} \mathrm{M}, 10^{-6} \mathrm{M}, 10^{-8} \mathrm{M}$ and $10^{-10} \mathrm{M}$, respectively. We have observed significant enhancement of the dye concentration of up to $10^{-10} \mathrm{M}$. The enhancement factor $(\mathrm{EF})$ value in SERS has been calculated according to previous equations ${ }^{5,56}$ and the values are summarized in Table 3, considering a dye concentration of $10^{-8} \mathrm{M}$ and $10^{-10} \mathrm{M}$. From the equation, $I_{\text {SERS }}$ and $I_{\mathrm{RS}}$ denote the intensity of enhanced SERS sample and from the blank dye molecule, while $C_{\mathrm{SERS}}$ and $C_{\mathrm{RS}}$ are the analyte concentration in the SERS experiment and the analyte concentration in the normal Raman experiment. From Table 3, we can see that the higher EF we observed was $1.7 \times 10^{7}$ while we used a R Be concentration of $10^{-10} \mathrm{M}$. We examined the EF value for the peak positions at 1292,1491 and $1614 \mathrm{~cm}^{-1}$. It is important to mention that there are only a couple of reports on SERS taking Os NPs which have been performed recently using R6G and $\mathrm{MB}$ as dye molecules. ${ }^{21,22}$ While they used R6G as a dye, the observed EF is $\sim 10^{4}$ only. ${ }^{21}$ In our recent study, we observed the highest EF $\sim 1.3 \times 10^{7}$ taking MB as dye molecules. ${ }^{22}$ So in our present study, taking $\mathrm{R}$ Be as a dye, we obtained the highest $\mathrm{EF}$ value $\left(\sim 1.7 \times 10^{7}\right)$ ever reported for Os NPs to date. We have compared the SERS effect with other two morphologies we prepared, and the results are presented in Fig. 7B. From Fig. 7B, curve a denotes the Raman spectra of only $\mathrm{R}$ Be $\left(10^{-3} \mathrm{M}\right)$ and cube b-d were the SERS spectra, while using Os spheres, chain-like networks and aggregated clusters, respectively. From Fig. 7B, it was confirmed that the enhancement was the highest in case of aggregated clusters and the order of EF was as follows: aggregated clusters $>$ chain-like networks $>$ spheres. The EF value observed was not only the highest considered for Os NPs, but also comparable or superior to other noble metal NPs like $\mathrm{Au}$ or $\mathrm{Ag}^{5,21,22,56,59}$ The different $\mathrm{EF}$ for different morphologies of Os NPs was expected as the Kneipp group reported earlier that the SERS intensity changes when the particles size and morphology vary. ${ }^{60}$ The highest EF in the case
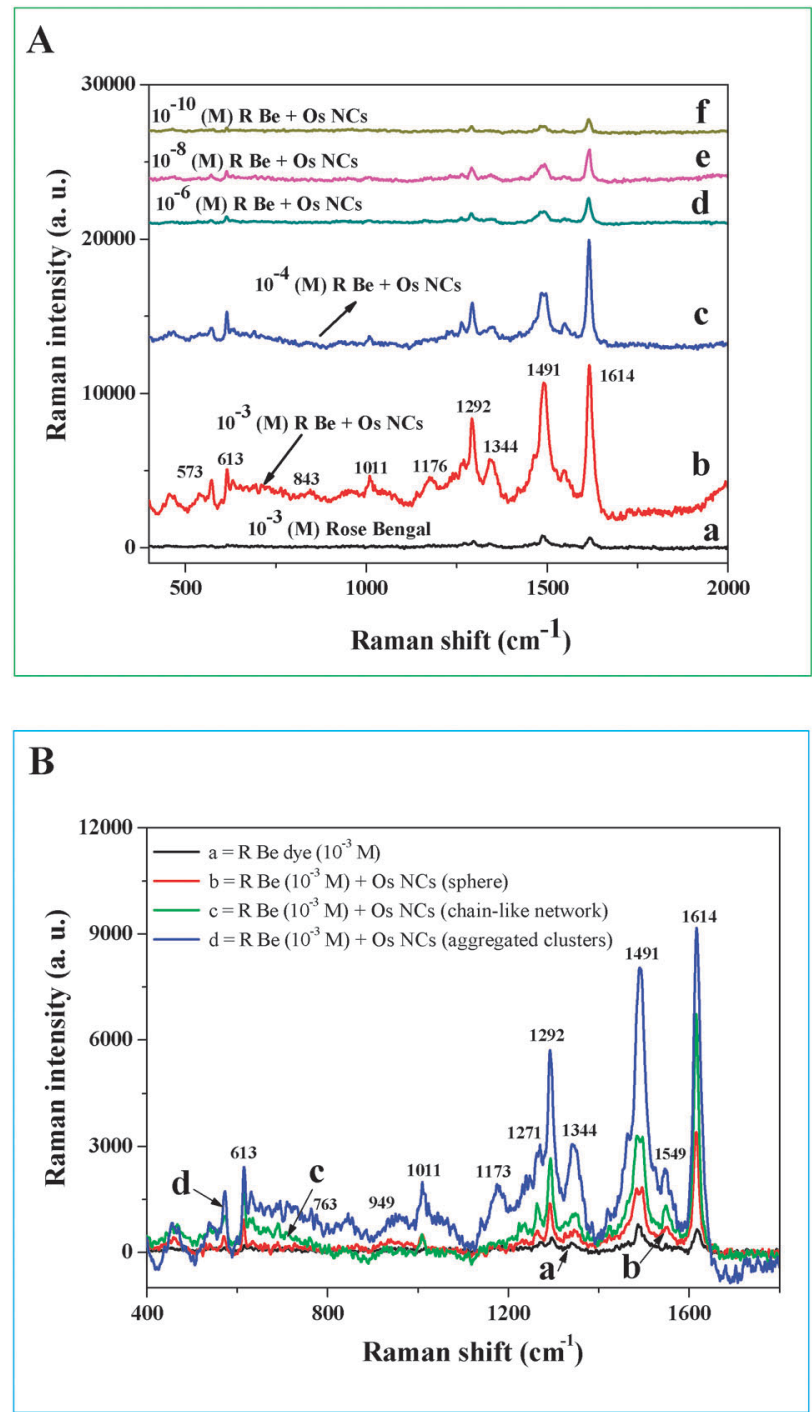

Fig. 7 (A) Surface enhanced Raman spectra (SERS) with different concentrations of R Be. Curve (a) shows Raman spectra of only $R$ Be dye at $10^{-3} \mathrm{M}$ concentration while curve $(b)-(f)$ show the SERS spectra at a dye concentration of $10^{-3} \mathrm{M}, 10^{-4} \mathrm{M}, 10^{-6} \mathrm{M}, 10^{-8} \mathrm{M}$ and $10^{-10} \mathrm{M}$, respectively. (B) Curve (a) denotes the Raman spectra of only R Be $\left(10^{-3} M\right)$ and curve (b)-(d) are the SERS spectra while using Os spheres, chain-like networks and aggregated clusters, respectively.

of aggregated clusters is probably due to generation of a greater number of active 'hot spots'. It was reported previously that when noble metal NPs aggregate, they provide a greater number of surface active 'hot spots' at the junction of the aggregates. ${ }^{54-57}$ The plasmonic coupling effect at those junctions or 'hot spots' creates a very high intensity local electromagnetic field and consequently leads to strong Raman cross-section enhancement. So we also believe that the self-assembled aggregated structure of Os NPs was responsible for the generation of a highly stable and reproducible SERS signal. To prove this phenomenon further, we ran a preliminary SERS study with the Os nano cube sample, as seen in Fig. S4B (ESI $\dagger$ ). We believe that the crystalline Os nanocube is generated due to over developed small spherical Os particles and the corresponding SERS result (not shown here) 
Table 3 Enhancement factor (EF) value in SERS for a dye concentration of $10^{-8} \mathrm{M}$ and $10^{-10} \mathrm{M}$

\begin{tabular}{|c|c|c|c|c|c|}
\hline \multirow[b]{2}{*}{ Nanoclusters (NCs) used } & \multirow[b]{2}{*}{ Dye used } & \multirow[b]{2}{*}{ Conc. of the dye used, $M$} & \multicolumn{3}{|c|}{ Enhancement factor (EF) values at a peak position } \\
\hline & & & $1292 \mathrm{~cm}^{-1}$ & $1491 \mathrm{~cm}^{-1}$ & $1614 \mathrm{~cm}^{-1}$ \\
\hline Os NCs (aggregated cluster) & $\begin{array}{l}\text { Rose Bengal } \\
\text { Rose Bengal }\end{array}$ & $\begin{array}{l}10^{-8} \\
10^{-10}\end{array}$ & $\begin{array}{l}6.95 \times 10^{5} \\
1.70 \times 10^{7}\end{array}$ & $\begin{array}{l}2.71 \times 10^{5} \\
8.45 \times 10^{6}\end{array}$ & $\begin{array}{l}6.31 \times 10^{5} \\
2.43 \times 10^{7}\end{array}$ \\
\hline
\end{tabular}

reveals that it also generates a high intensity SERS signal with a higher EF value. Moreover, it was interesting to say that similar results were observed in a catalysis reaction where we observed the highest catalytic rate in the case of an aggregated cluster structure as compared to others, although the mechanism of the catalysis reaction is somewhat different from the SERS experiment. A more detailed experimental and theoretical study is necessary to get an in-depth idea of each parameter contributing to the enhancement, and will be discussed in near future.

\section{Conclusions}

In summary, we report a new route for the formation of osmium NCs having different morphologies by utilizing a simple wet-chemical route at room temperature. The Os NCs having aggregated clusters, chain-like networks, and small spheres were prepared by a reaction of $\mathrm{OsO}_{4}$, CTAB, 2,7-DHN, and $\mathrm{NaOH}$ under continuous stirring for a total of $30 \mathrm{~min}$. The morphology of the particles can be changed by tuning the Os salt to the CTAB molar ratio and varying other reaction parameters. The synthesized materials have been tested for catalysis and SERS studies. The catalysis study was examined with different organic nitro compounds. The observed catalysis reaction rate was found superior as compared to other noble metal NPs. The SERS study was performed taking Rose Bengal (R Be) as a probe molecule and the observed EF value was found superior or comparable to most of the other noble metals NPs. The morphological effect of Os NCs has also been tested and it was found that Os NCs having aggregated clusters give better results both in a catalysis and in a SERS study. The overall synthesis process was simple, fast and cost-effective. The enhanced catalytic and SERS activities of the material might opens up a new avenue for application in other organic catalysis reactions, SERS based detection of environmentally important biomolecules and sensors.

\section{Acknowledgements}

S. K. wishes to thank Dr V. K. Pillai, Director and Dr M. Jayachandran, HOD-ECMS Division, CSIR-CECRI for their support and encouragement. S. R. E. and U. N. wish to acknowledge CSIR for JRF fellowship and CECRI for research internship fellowship, respectively. Support from the Central Instrumental Facility (CIF) and help from Mr A. Rathishkumar (TEM in-charge, CIF), Mr R. Ravishankar and Mr J. Kennedy (SEM in-charge, CIF), CSIR-CECRI, Karaikudi are greatly appreciated. S. Kundu wishes to acknowledge CSIR-CECRI for start-up research funding (Project number IHP 0067, DU No. 5, old number OLP-0067).

\section{References}

1 R. Gill, R. F. Saraf and S. Kundu, ACS Appl. Mater. Interfaces, 2013, 5, 9949.

2 S. Kundu, S. Lau and H. Liang, J. Phys. Chem. C, 2009, 113, 5150.

3 S. Kundu, M. D. Mukadam, S. M. Yusuf and M. Jayachandran, CrystEngComm, 2013, 15, 482.

4 F. Trager, Appl. Phys. B: Lasers Opt., 2001, 73, 291.

5 S. Kundu, J. Mater. Chem. C, 2013, 1, 831.

6 S. Kundu and H. Liang, Adv. Mater., 2008, 20, 826.

7 C. N. R. Rao, G. U. Kulkarni, P. J. Thomas and P. P. Edwards, Chem. Soc. Rev., 2000, 29, 27.

8 M. B. Gawande, S. N. Shelke, R. Zboril and R. S. Varma, Acc. Chem. Res., 2014, 47, 1338.

9 K. E. Sapsford, W. R. Algar, L. Berti, K. B. Gemmill, B. J. Casey, E. Oh, M. H. Stewart and I. L. Medintz, Chem. Rev., 2013, 113, 1904.

10 M. B. Gawande, P. S. Brancoa and R. S. Varma, Chem. Soc. Rev., 2013, 42, 3371.

11 CRC Handbook of Chemistry and Physics, ed. W. M. Haynes, CRC Press/Taylor and Francis, Boca Raton, FL, 2011, 91st edn (Internet Version 2011).

12 E. P. Boyd, D. R. Ketchum, H. Deng and S. G. Shore, Chem. Mater., 1997, 9, 1154.

13 A. Forleo, S. Capone, M. Epifani, P. Siciliano and R. Rella, Appl. Phys. Lett., 2004, 84, 744.

14 K.-F. Yung and W.-T. Wong, J. Cluster Sci., 2007, 18, 51.

15 T. Jones, Met. Finish., 2002, 100, 84.

16 F. Quaranta, R. Rella, P. Siciliano, S. Capone, M. Epifani, L. Vasanelli, A. Licciulli and A. Zocco, Sens. Actuators, B, 1999, 58, 350.

17 V. W. S. Lam and E. L. Gyenge, J. Electrochem. Soc., 2008, 155, B1155.

18 E. Borja-Arco, R. H. Castellanos, J. Uribe-Godínez, A. AltamiranoGutiérrez and O. Jiménez-Sandoval, J. Power Sources, 2009, 188, 387.

19 J. Kramer, E. Redel, R. Thomann and C. Janiak, Organometallics, 2008, 27, 1976.

20 J. Hamalainen, T. Sajavaara, E. Puukilainen, M. Ritala and M. Leskala, Chem. Mater., 2012, 24, 55.

21 K. Chakrapani and S. Sampath, Chem. Commun., 2013, 49, 6173.

22 U. Nithiyanantham, S. R. Ede and S. Kundu, J. Mater. Chem. C, 2014, 2, 3782 .

23 N. Pradhan, A. Pal and T. Pal, Langmuir, 2001, 17, 1800. 
24 N. Tian, Z.-Y. Zhou, S.-G. Sun, Y. Ding and Z. L. Wang, Science, 2007, 316, 732.

25 M. Chirea, A. Freitas, B. S. Vasile, C. Ghitulica, C. M. Pereira and F. Silva, Langmuir, 2011, 27, 3906.

26 S. Kundu, K. Wang and H. Liang, J. Phys. Chem. C, 2009, 113, 5157.

27 S. R. Emory, W. E. Haskins and S. Nie, J. Am. Chem. Soc., 1998, 120, 8009.

28 M. Moskovits, J. Raman Spectrosc., 2005, 36, 485.

29 J. P. Camden, J. A. Dieringer, Y. Wang, D. J. Masiello, L. D. Marks, G. C. Schatz and R. P. Van Duyne, J. Am. Chem. Soc., 2008, 130, 12616.

30 S. Kundu and U. Nithiyanantham, RSC Adv., 2013, 3, 25278. 31 W. R. Mason III and H. B. Gray, Inorg. Chem., 1968, 7, 55.

32 J. A. Creighton and D. G. Eadon, J. Chem. Soc., Faraday Trans., 1991, 87, 3881.

33 J. J. Storhoff, A. A. Lazarides, R. C. Mucic, C. A. Mirkin, R. L. Letsinger and G. C. Schatz, J. Am. Chem. Soc., 2000, $122,4640$.

34 C. H. Lee, K. Lee, S. Chang, J. T. Park and B. Kim, Korean Chem. Soc., 2006, 27, 130.

35 S. Honary, H. Barabadi, E. Gharaei-Fathabad and F. Naghibi, Trop. J. Pharm. Res., 2013, $12,7$.

36 Y. Wang, L. Q. Chen, Y. F. Li, X. J. Zhao, L. Peng and C. Z. Huang, Nanotechnology, 2010, 21, 305601.

37 S. Kundu, K. Wang and H. Liang, J. Phys. Chem. C, 2009, 113, 134.

38 A. Pal and T. Pal, J. Raman Spectrosc., 1999, 30, 199.

39 T. Sato, N. Maeda, H. Ohkoshi and Y. Yonezawa, Bull. Chem. Soc. Jpn., 1994, 67, 3165.

40 A. Henglein, Langmuir, 1999, 15, 6738.

41 K. Esumi, A. Suzuki, N. Aihara, K. Usui and K. Torigoe, Langmuir, 1998, 14, 3157.

42 S. Kundu, A. Pal, S. K. Ghosh, S. Nath, S. Panigrahi, S. Praharaj and T. Pal, Inorg. Chem., 2004, 43, 5489.
43 S. Kundu, Phys. Chem. Chem. Phys., 2013, 15, 14107.

44 S. Kundu and M. Jayachandran, RSC Adv., 2013, 3, 16486.

45 N. R. Jana, L. Gearheart and C. J. Murphy, J. Phys. Chem. B, 2001, 105, 4065.

46 Y. Song, R. M. Garcia, R. M. Dorin, H. Wang, Y. Qiu, E. N. Coker, W. A. Steen, J. E. Miller and J. A. Shelnutt, Nano Lett., 2007, 7, 3650.

47 T. Hardar, R. Stober, P. Wessig and J. Bendig, J. Photochem. Photobiol., A, 1997, 103, 105.

48 A. Saha and B. Ranu, J. Org. Chem., 2008, 73, 6867.

49 Y. Lu, Y. Mei, M. Drechsler and M. Ballauff, Angew. Chem., Int. Ed., 2006, 45, 813.

50 R. Vadakkekara, M. Chakraborty and P. A. Parikh, Colloids Surf., A, 2012, 399, 11.

51 K. S. Shin, J. H. Kim, I. H. Kim and K. Kim, Bull. Korean Chem. Soc., 2012, 33, 906.

52 M. Fleischmannn, P. J. Hendra and A. J. MacQuillan, Chem. Phys. Lett., 1974, 26, 163.

53 C. G. Blatchford, J. R. Campbell and J. A. Creighton, Surf. Sci., 1982, 120, 435.

54 B. Nikoobakht and M. A. El-Sayed, J. Phys. Chem. A, 2003, 107, 3372.

55 V. A. Markel, V. M. Shalaev, P. Zhang, W. Huynh, L. Tay, T. L. Haslett and M. Moskovits, Phys. Rev. B: Condens. Matter Mater. Phys., 1999, 59, 10903.

56 A. M. Michaels, J. Jiang and L. Brus, J. Phys. Chem. B, 2000, 104, 11965.

57 D. Majumdar, A. Singha, P. K. Mondal and S. Kundu, ACS Appl. Mater. Interfaces, 2013, 5, 7798.

58 A. M. Gabudean, M. Focsan and S. Astilean, J. Phys. Chem. C, 2012, 116, 12240.

59 Y. Xiong, J. M. McLellan, J. Chen, Y. Yin, Z. Y. Li and Y. N. Xia, J. Am. Chem. Soc., 2005, 127, 17118.

60 K. Kneipp, H. Kneipp, I. Itzkan, R. R. Dasari and M. S. Feld, Chem. Rev., 1999, 99, 2957. 